\title{
Fatores que influenciam a prática do profissional de saúde no manejo da dor do recém-nascido
}

\author{
Factors that influence the practice of healthcare professionals regarding pain management \\ in newborn infants
}

Alessandra Costa A. Maia', Sônia Bechara Coutinho²

\section{RESUMO}

Objetivo: Apresentar revisão sobre as principais variáveis que podem influenciar as ações e as atitudes dos profissionais de saúde no manejo adequado da dor no período neonatal.

Fontes dos dados: Pesquisa bibliográfica, nas bases de dados Medline, SciELO e Lilacs, entre 1995 e 2009, restrita aos artigos escritos em inglês e português. Foram utilizados os seguintes descritores: "dor", "Terapia Intensiva Neonatal" e "profissional de Saúde”. Avaliaram-se 88 artigos, sem seleção pelo desenho, sendo incluídos os 55 mais relevantes e recentes.

Síntese dos dados: Os estudos confirmaram que o manejo da dor no neonato pelos profissionais de saúde é inadequado. Essa prática pode estar relacionada aos seguintes fatores: conhecimento insuficiente quanto à fisiopatologia da dor; não utilização de métodos de avaliação; falta de normatização sistemática ou protocolos; e falha na comunicação e incorporação da teoria na prática diária por parte da equipe multiprofissional. São acrescidos ainda os fatores subjetivos, pessoais e profissionais, que podem interferir na avaliação e no tratamento da dor no recém-nascido.

Conclusões: Os obstáculos para um manejo adequado da dor são multifatoriais e o conhecimento das atitudes e crenças das equipes de saúde que trabalham com neonatos é muito importante. Há necessidade de investimentos na capacitação, formação e sensibilização dos profissionais acerca do controle e do tratamento da dor, com o intuito de proporcionar um cuidado mais humanizado ao neonato.

Palavras-chave: dor; terapia intensiva neonatal; pessoal de Saúde.

\section{ABSTRACT}

Objective: To perform a literature review on the main variables associated with in the actions and attitudes of healthcare professionals regarding pain management during the neonatal period.

Data sources: Bibliographic search of Medline, SciELO and Lilacs databases for articles published in English and Portuguese between 1995 and 2009, using the descriptors "pain", "Neonatal Intensive Care" and "health personnel". Among 88 articles, the 55 more recent and relevant ones were included.

Data synthesis: The studies confirmed that neonatal pain management by healthcare professionals is inadequate. This may be related to insufficient knowledge regarding the physiopathology of pain; lack of systematic guidelines on pain assessment and management and failure in communication and incorporation of knowledge into the clinical practice by health professionals. Moreover, there are individual and professional subjective factors that may affect pain evaluation and treatment in newborns.

Conclusions: There are multiple obstacles to adequate neonatal pain management. Understanding the attitudes and beliefs of healthcare teams is important to assess and heat neonatal pain. There is a need for investments on the qualification and awareness of healthcare professionals regarding pain control in order to provide humanized neonatal care.

Key-words: pain; intensive care, neonatal; Health personnel.
Instituição: Universidade Federal de Pernambuco (UFPE), Recife, PE, Brasil

${ }^{1}$ Mestre pelo do Programa de Saúde da Criança e do Adolescente da UFPE, Recife, PE, Brasil

2Doutora em Nutrição pela UFPE; Professora do Departamento MaternoInfantil e da Pós-graduação em Saúde da Criança e do Adolescente da UFPE, Recife, PE, Brasil
Endereço para correspondência:

Alessandra Costa A. Maia

Rua Ferreira Lopes, 401/2001 - Casa Amarela

CEP 52060-200 - Recife/PE

E-mail: doca_maia@yahoo.com.br

Conflito de interesse: nada a declarar 


\section{Introdução}

Nos últimos anos, avanços importantes foram obtidos no entendimento da nocicepção em recém-nascidos. Desde 1970, os estudos têm demonstrado a existência do substrato neuroquímico necessário para a recepção e a transmissão do estímulo doloroso em crianças e recém-nascidos ${ }^{(1,2)}$. Apesar disso, a dor no neonato não tem merecido a atenção devida nos serviços de saúde ${ }^{(3)}$.

Os estímulos dolorosos repetidos no período neonatal resultam em consequências negativas sob a forma de aumento na mortalidade, sequelas no desenvolvimento neurológico e somatização anormal frente à dor em outras fases da vida ${ }^{(4,5)}$. O comportamento e a percepção da dor em longo prazo são alterados diante da dor persistente e de caráter repetitivo ${ }^{(6)}$. Como consequência, a diminuição da exposição a eventos álgicos ou estressantes pode levar a resultados melhores, tanto clínicos, como no desenvolvimento neurológico do neonato ${ }^{(2,7)}$. Os estudos têm demonstrado que, apesar do conhecimento crescente sobre a dor e o avanço no seu tratamento, o uso de analgesia de rotina, no período neonatal, para procedimentos dolorosos, ainda é insuficiente e inadequado ${ }^{(8-}$ 11). A prescrição restrita de fármacos pelos neonatologistas pode ser justificada pela falta de opções terapêuticas seguras e efetivas, possibilidade de efeitos adversos, com evidências insuficientes para sua utilização de forma prolongada ${ }^{(12,13)}$.

A dificuldade para avaliar e mensurar a dor no lactente pré-verbal constitui um dos maiores obstáculos ao seu tratamento nas Unidades de Terapia Intensiva Neonatal (UTIN) ${ }^{(14)}$ e os profissionais mostram-se, quase sempre, pouco capazes de identificar e tratar a dor do recém-nascido ${ }^{(3,15,16)}$. Nesse processo, o principal objetivo é o alívio do fenômeno álgico, sendo a avaliação o primeiro passo para o manejo adequado da dor ${ }^{(17)}$. No entanto, a maioria das escalas de dor estuda o comportamento das crianças em situações de dor aguda, por meio de instrumentos de medida unidimensionais, como a expressão de manifestações fisiológicas e comportamentais, mais facilmente observáveis na dor aguda quando comparada à crônica ${ }^{(18)}$.

Os indicadores da dor passíveis de observação podem ser mínimos ou ausentes, o que exige a adequação na forma de avaliação da dor por parte dos profissionais de saúde, no sentido de maior sensibilização para atender as necessidades dos cuidados dos recém-nascidos ${ }^{(19-21)}$, utilizando os conhecimentos científicos em sua rotina ${ }^{(22,23)}$. Observa-se uma lacuna entre a teoria e a conduta clínica da equipe de saúde, que pode ser devida, entre outros, a falhas na incorporação da teoria na prática diária, a fatores inerentes ao neonato e a fatores relacionados aos profissionais, como aspectos pessoais, emocionais e habilidade de trabalhar com equipe interdisciplinar $^{(14,16,23-28)}$.

Os obstáculos para um manejo adequado da dor no neonato são multifatoriais e devem ser analisados como um processo dinâmico e interativo entre a criança e o seu cuidador, conforme estabelecido no modelo sociocomunicativo da dor na infância ${ }^{(29)}$. Com base nessa estruturação, foi idealizado o modelo conceitual do manejo adequado da dor pelo profissional de saúde, tendo como principais pontos que se interrelacionam o trinômio expressão da dor do recémnascido, experiência pessoal e profissional em lidar com a dor e engajamento da instituição.

Dessa forma, a finalidade deste artigo de revisão foi discutir as variáveis que podem influenciar ou interferir nas ações e atitudes dos profissionais em sua prática clínica. Para abordagem do tema, foi revista a literatura relacionada, utilizando as bases de dados Medline, SciELO e Lilacs, no período de 1995 a 2009, com artigos escritos em inglês e português. Foram selecionados os descritores: "dor", "Terapia Intensiva Neonatal" e "profissional de saúde". Foram avaliados 88 artigos, sem seleção pelo desenho, priorizando aqueles mais relevantes e recentes, sendo incluídos 55 deles (22 estudos transversais, 14 revisões da literatura, seis estudos prospectivos, um caso-controle, um retrospectivo, três ensaios clínicos, três consensos e cinco qualitativos).

\section{Expressão da dor do neonato}

Fatores inerentes ao paciente, como idade gestacional, sexo, raça, aparência física, presença de dano tecidual e gravidade do diagnóstico podem alterar a inferência da presença e magnitude de dor, pelo observador ${ }^{(26,30-33)}$. Em relação ao aspecto físico e à maturidade do neonato, as pesquisas evidenciam que os recém-nascidos de maior peso e com idade gestacional mais elevada recebem analgesia com mais frequência ${ }^{(22,34)}$. Na literatura, porém, já está estabelecido que neonatos prematuros são altamente sensíveis à dor, e necessitam de doses relativas maiores de anestésicos e analgésicos para o seu controle adequado ${ }^{(5,35)}$. Outros estudos demonstram que o observador reconhece melhor a presença e a intensidade do fenômeno doloroso na criança do sexo feminino ${ }^{(30,32,33,36)}$. Além disso, os pacientes debilitados e sem boa aparência física podem induzir o profissional a diagnosticar a presença e a intensidade da dor mais frequentemente ${ }^{(37)}$. 
No processo clínico de tomada de decisões, no qual o objetivo é o alívio da dor, o primeiro passo é uma avaliação ampla e adequada da experiência dolorosa ${ }^{(20)}$. Contudo, no período neonatal, por envolver pacientes naturalmente vulneráveis e dependentes de seus cuidadores, o processo doloroso está intrinsecamente relacionado às condutas dos profissionais de saúde e tal dependência constitui um dos maiores obstáculos ao tratamento adequado da dor, pela equipe de saúde, nas UTIN $^{(16,22,30,38)}$.

Para lograr objetividade, foram desenvolvidas várias escalas de avaliação da dor. Essas escalas, porém, ainda não foram implementadas na prática clínica da maioria das UTIN $^{(14,22,34)}$. Pesquisadores demonstram preocupação ao observar que médicos e enfermeiras não as têm utilizado nas rotinas dos serviços ${ }^{(8,22,23)}$. Um estudo realizado em nosso meio, em 2006, com enfermeiras que trabalham com recém-nascidos de risco internados em unidades neonatais, observou que apenas um quarto das mesmas referiram ter conhecimento sobre algum tipo de escala para avaliar a dor em neonatos ${ }^{(39)}$. Da mesma forma, Bergqvist $e t$ al, em estudo publicado em 2007, concluíram que os neonatologistas têm dificuldades em avaliar a presença e a intensidade da dor no recém-nascido ${ }^{(40)}$. Algumas das justificativas para tais dificuldades são: falta de treinamento dos profissionais, necessidade de escalas que avaliem a dor do recém-nascido de diferentes idades gestacionais - especialmente prematuros extremos, e inexistência de uma escala considerada padrão-ouro para a mensuração da dor nessa faixa etária ${ }^{(19,21)}$.

\section{Experiência do profissional em lidar com a dor}

Diversos fatores, como idade, sexo, raça, religião, estado civil, nível socioeconômico e tradições culturais, podem influenciar a avaliação da dor neonatal pelos profissionais de saúde ${ }^{(18,31,35,36,41)}$. A influência da idade do avaliador na percepção do fenômeno doloroso do neonato é um tema controverso $^{(36)}$. Porter $e^{2} a^{(35)}$, ao avaliarem a equipe médica e de enfermagem, observaram que os médicos mais idosos ou que trabalhavam em enfermarias de maior complexidade relataram maior uso de agentes farmacológicos. Quanto ao gênero do avaliador, estudos clínicos têm demonstrado sua influência na percepção da dor, sendo influenciado por variáveis psicológicas e, no caso das mulheres, parece estar relacionado a critérios mais brandos para a avaliação da dor ${ }^{(31)}$. Como consequência, já foram relatadas divergências quanto ao gênero feminino e ao masculino no tratamento clínico da dor, considerando que os profissionais do gênero feminino avaliam a dor com mais intensidade ${ }^{(32)}$.

A forma de avaliar a resposta à dor pode ser influenciada pelas diferenças culturais e raciais dos observadores ${ }^{(36,42)}$. Dois estudos que utilizaram um mesmo questionário para avaliar o nível de aceitação social diante de várias maneiras de expressar a dor em grupos étnicos diferentes demonstraram que os estudantes indianos e japoneses aceitavam menos as expressões públicas de dor, quando comparados aos americanos $^{(31,42)}$. Esses resultados podem ser explicados pelo estoicismo e pelo conformismo religioso de uma sociedade etnicamente homogênea, educada para se resignar e esconder suas dores e emoções, em oposição à cultura euroamericana pluralista, mais permissiva e liberal, que enfatiza a expressão de seus sentimentos. Além disso, a administração de analgésicos pode ser influenciada por preconceitos sociais, como demonstrado em pesquisa americana, que observou menor prescrição de tratamento específico, pelos médicos da emergência, aos pacientes de origem hispânica ${ }^{(43)}$. Por essa razão, a equipe de saúde precisa estar atenta quanto ao viés cultural e de gênero, para não haver influência na decisão do tratamento ${ }^{(31)}$.

Uma pesquisa que entrevistou profissionais de saúde e pais de recém-nascidos que trabalhavam em outras áreas de atuação, realizada com o objetivo de avaliar o reconhecimento de expressões de dor em neonatos, verificou que o reconhecimento da expressão de dor se associou a algumas características dos entrevistados, como: união estável, ter mais de duas crianças e não ser profissional da área de saúde ${ }^{(36)}$.

A relação de tempo de experiência em neonatologia com a melhora do manejo da dor ainda é assunto controverso ${ }^{(23,35,39)}$. Porém, para a maioria dos pesquisadores, os especialistas avaliam com mais dificuldade os procedimentos dolorosos, quando comparados aos generalistas. A maior bagagem educacional, e a experiência profissional mais especializada e duradoura, além do convívio mais próximo com a dor de pacientes, podem dificultar a percepção da dor no recémnascido ${ }^{(14,30)}$. Apesar do conhecimento e da experiência clínica, os especialistas não as transferem para a prática ${ }^{(26)}$. Os profissionais que realizam procedimentos invasivos de modo rotineiro diminuem sua sensibilidade por meio de uma reestruturação cognitiva, tornando-se mais céticos em relação às respostas subjetivas de dor exibidas pelas crianças. Os resultados, então, podem refletir uma insensibilidade ao fenômeno clínico associado à dor neonatal ou podem resultar de uma avaliação baseada na "doença" e não no "doente"(40). 
A percepção de dor por profissionais de saúde, que subsequentemente tomam a decisão sobre como tratá-la, não é um processo simples e pode ser influenciada por uma série de fatores relacionados às outras características dos próprios observadores, como a experiência prévia, pessoal ou familiar, com a dor ${ }^{(28,30,31,36)}$. Um estudo brasileiro realizado com enfermeiros que atendem crianças internadas em UTI demonstrou que a avaliação da dor da criança estava entremeada pela sensibilidade, na medida em que o profissional revive a própria experiência dolorosa ou a de seu filho. Com essa vivência, passa a valorizar a situação dolorosa do recém-nascido e aumenta consideravelmente a administração de analgésicos em situações clínicas ${ }^{(44)}$. Médicos que relataram passado cirúrgico ou experiência pessoal com dor classificaram melhor os procedimentos dolorosos, utilizaram mais vezes analgesia farmacológica e indicavam com maior frequência as medidas de alívio da dor não farmacológicas ${ }^{(32)}$. Robinson e Wise ${ }^{(35)}$ analisaram a influência de uma experiência dolorosa prévia dos profissionais sobre a sua avaliação de fenômeno álgico. Os autores verificaram que a dor, de forma geral, foi subestimada, porém o grupo que posteriormente sofreu a mesma experiência dolorosa dos avaliados mudou sua percepção do fenômeno álgico e apresentou mais sensibilidade para diagnosticar a dor ${ }^{(32)}$. Dessa forma, a percepção da dor do paciente e a motivação para indicar o alívio da mesma são influenciadas pela relação interpessoal médico/paciente, na qual o médico, a partir do sofrimento do paciente, reativa vários sentimentos de sua vida, o que pode interferir na sua conduta frente aos fenômenos dolorosos ${ }^{(45)}$.

Quando os profissionais são avaliados em conjunto, tanto médicos como enfermeiros acreditam que o uso de agentes farmacológicos e medidas de alívio para a dor deveriam ser usados mais frequentemente ${ }^{(35,46,47)}$. Apesar do conhecimento atual de que o prematuro é capaz de sentir dor, medidas ambientais e/ou farmacológicas não são utilizadas com frequência, nas situações dolorosas, em pacientes internados na UTIN ${ }^{(11,25,35)}$. O temor pode ser justificado pela falta de opções terapêuticas seguras e efetivas que permitam sua utilização de forma prolongada ${ }^{(12,13)}$.

A morfina e o fentanil são os opioides mais usados em recém-nascidos, sendo a primeira citada como fármaco de escolha segundo os consensos mundiais para o tratamento da dor nessa população ${ }^{(48)}$. Porém a indicação da morfina vem sendo criticada nos prematuros extremos e nos pacientes em uso de ventilação mecânica, sendo a hipotensão e a depressão respiratória efeitos adversos observados, com aumento do tempo de uso do respirador mecânico pelos recém-nascidos que a utilizam ${ }^{(12,13)}$. Com relação ao fentanil, é um fármaco muito utilizado na neonatologia devido à sua capacidade de analgesia rápida, com estabilidade hemodinâmica ${ }^{(8)}$. Já o diazepínico, ainda que comumente usado, é um sedativo que age diminuindo a atividade, a ansiedade e a agitação do paciente, porém não possui ação analgésica e deve ser usado de forma criteriosa ${ }^{(16,48)}$. É importante salientar que as medidas não farmacológicas são importantes, mas não substituem o tratamento medicamentoso nos casos de dor mais intensa ${ }^{(49)}$.

A preocupação com a superdosagem, a dependência e a depressão respiratória tem sido citada como razão para os cuidadores relutarem na administração de analgésicos e anestésicos ${ }^{(12)}$. Conforme observado em um estudo com enfermeiras do Reino Unido, o manejo inadequado da dor é mais um reflexo do desconhecimento propriamente dito do que de influências de vieses ou mitos ${ }^{(26)}$. Assim, é necessário que os médicos que realizam procedimentos nos neonatos estejam familiarizados com o manejo da dor. Isso inclui atualização constante sobre os agentes farmacológicos, dose específica para a idade, via de administração apropriada e os possíveis efeitos adversos ${ }^{(50)}$.

Em relação ao distanciamento entre o conhecimento e a prática, o primeiro tem avançado consideravelmente nas últimas duas décadas, com maior entendimento dos mecanismos neurobiológicos da dor, bem como da frequência e da natureza dos procedimentos dolorosos nas UTIN $^{(2,5)}$. Porém, muitas vezes, as atitudes e práticas dos profissionais, no que concerne ao manejo adequado da dor no período neonatal, não refletem os atuais conhecimentos científicos para a faixa etária ${ }^{(15,22,23)}$. Em estudo recente realizado em duas UTIN norueguesas, que descreveu a opinião dos profissionais de saúde sobre procedimentos dolorosos em neonatos, verificou-se que as respostas indicaram diferença significativa entre o tratamento de rotina utilizado e o preconizado. Tanto as medidas de conforto como os agentes farmacológicos foram subutilizados nos serviços estudados ${ }^{(46)}$.

A maioria das enfermeiras londrinas, quando avaliadas por meio de questionário, referiu não utilizar escalas de avaliação de dor e grande parte relatou não usar analgésico para procedimentos dolorosos, com exceção de inserção de dreno torácico, circuncisão e intubação traqueal. Uma das entrevistadas comentou: "Quando o procedimento ocorre por todo o dia é impraticável oferecer analgesia continuamente"(15). 
Em pesquisa realizada no Município de Belém, no Estado do Pará, com médicos pediatras que trabalhavam em UTIN e/ou berçários de baixo e médio risco, foi observado que, apesar de todos os médicos referirem que o neonato sente dor e reconhecerem a importância do seu tratamento, mais da metade dos profissionais afirmou não usar medicação para o tratamento antiálgico no pós-operatório imediato de cirurgia abdominal ${ }^{(14)}$. Nessa mesma linha, estudo realizado em UTIN de São Paulo verificou que as escalas não foram utilizadas em nenhum paciente submetido à ventilação pulmonar mecânica ${ }^{(22)}$. Resultado semelhante foi constatado em estudo multicêntrico italiano, em 2005, que mostrou a utilização de escalas validadas para a avalição da dor em menos de $20 \%$ das UTIN do país ${ }^{(8)}$.

Um estudo qualitativo com enfermeiros, na cidade de Oslo, evidenciou discordância entre o que foi afirmado na entrevista dos profissionais e as ações na prática de rotina, em relação ao manejo da dor no pós-operatório. Essa situação pode ser devida ao conhecimento inadequado ou insuficiente sobre a definição e o manejo da dor, à não incorporação do procedimento ao plano pessoal e ao seguimento de práticas e hábitos tradicionais, sem vinculação das próprias experiências ${ }^{(26)}$. Outro ponto que pode justificar a não utilização da teoria preconizada é a alta rotatividade dos profissionais nas unidades neonatais, havendo necessidade de capacitação continuada da equipe, principalmente dos recém-formados e daqueles com menor experiência na área de atuação ${ }^{(24)}$.

Pesquisa para verificar os conhecimentos e as práticas sobre atenção humanizada aos recém-nascidos de baixo peso observou que poucos profissionais de saúde relataram o uso de fármacos durante procedimentos sabidamente dolorosos ${ }^{(27)}$. Dessa forma, existe um lapso entre o conhecimento teórico e a conduta clínica, que pode ser devido a falhas na incorporação da teoria a respeito do diagnóstico e do tratamento da dor à prática profissional diária ${ }^{(14,16,23-27)}$.

\section{Engajamento da instituição}

A comunicação entre os profissionais de saúde sobre a presença ou a ausência da dor no recém-nascido no cotidiano da UTIN deve abranger o conjunto da equipe, com a finalidade de que todos estejam atentos para os fatores desencadeantes e as medidas de controle do processo álgico. Estudos demonstram que um dos obstáculos para o manejo e o tratamento adequado da dor é a falha na comunicação entre enfermeiros, médicos e pacientes quanto à sua avaliação e ao seu tratamento ${ }^{(26,28,47)}$.
A importância da interdisciplinaridade no manejo dos processos dolorosos está bem estabelecida, porém, na prática clínica, ela ocorre muito pouco ${ }^{(15,24)}$. Na maioria das vezes, é estabelecida uma rotina na qual as medidas não farmacológicas de alívio da dor são de responsabilidade da enfermagem e os médicos limitam-se ao manejo da dor com a perspectiva farmacológica ${ }^{(35,46)}$. Como relatado em estudo qualitativo com enfermeiras que atendem a crianças internadas na UTI, um dos obstáculos enfrentados para o adequado manejo da dor é a resistência à interação com os demais profissionais da equipe de saúde ${ }^{(44)}$.

O fluxo de comunicação deve ser estabelecido entre todos os componentes da equipe. Se a dor for identificada, mas a informação não circular entre os profissionais ou circular de modo restrito, irregular e lento, a proposta analgésica e seus ajustes ficam comprometidos ${ }^{(47)}$.

Outro fator institucional a ser levado em conta é a falta de normatização e protocolos. O emprego de protocolos que facilitem a seleção correta de medicamentos, sua administração adequada e a monitorização cuidadosa pode melhorar a qualidade da sedação e da analgesia, além de efeitos adversos ${ }^{(28,47,51)}$. Por essa razão, alguns consensos internacionais que estabelecem normatizações para o manejo adequado da dor neonatal foram publicados ${ }^{(48,52,53)}$.

Estudos multicêntricos recentes revelaram que apenas uma minoria das instituições usa escalas ou protocolos escritos para o manejo da dor ${ }^{(8,34)}$. Poucos serviços possuem uma política ou normatização escrita para o manejo prático da dor durante os procedimentos e, na maioria dos hospitais, não há registro, no prontuário, do uso de escalas ou tratamento analgésico ${ }^{(9,10,34)}$. Entretanto, a presença de normatizações escritas pode melhorar a atenção à dor nas UTIN, conforme demonstrado em estudo europeu em que a avaliação do fenômeno doloroso e o uso de terapia analgésica foram mais frequentes nos serviços em que existiam normatizações para o manejo da dor no período neonatal ${ }^{(34)}$.

Neste contexto, em um estudo no Reino Unido, foram recomendadas a todas as unidades neonatais do país normatizações escritas sobre analgesia nas UTIN, tornando-as disponíveis a todos os profissionais, principalmente aos recémformados ${ }^{(10)}$. Recomendações semelhantes foram destacadas em pesquisa neozelandesa que, após seis anos, transformou as evidências científicas em prática ${ }^{(54)}$. Uma base ampla de conhecimentos e atualização relacionada ao manejo e à avaliação da dor é primordial para auxiliar os profissionais no tratamento dos recém-nascidos que necessitam de cuidados intensivos ${ }^{(48)}$. 


\section{Conclusões e recomendações}

Segundo o consenso para o manejo e a prevenção da dor no neonato, o profissional de saúde tem a responsabilidade de avaliar, prevenir e manejar adequadamente a dor em recém-nascidos ${ }^{(53)}$. Os motivos que justificam a pouca utilização de analgesia pelos profissionais em suas rotinas ainda não são claros, apesar dos estudos demonstrarem os benefícios da analgesia para o paciente ${ }^{(10)}$. As dificuldades perpassam por aspectos cognitivos e emocionais do profissional até a falta de opções terapêuticas eficazes e que forneçam analgesia de forma segura.

Uma abordagem multidisciplinar nas UTIN, para o manejo da dor, pode melhorar a assistência ao recém-nascido ${ }^{(3)}$. Estudos referem que a falta de comunicação entre a equipe de saúde e a pouca organização das unidades dificultam a avaliação e tratamento da dor. A influência de fatores pessoais, o pouco entendimento entre os profissionais, as dificuldades de comunicação com os familiares e a falta de

\section{Referências bibliográficas}

1. Guinsburg R, Kopelman BI, Almeida MF, Miyoshi MH. Pain in intubated and ventilated preterm neonate: multidimensional assessment and response to fentanyl analgesia. J Pediatr (Rio J) 1994;70:82-90.

2. Hall RW, Anand KJ. Short and long-term impact of neonatal pain and stress: more than an ouchie. Neoreviews 2005;6:69-75.

3. Gaíva MA, Dias NS. Dor no recém-nascido: percepção de profissionais de saúde de um hospital universitário. Rev Paul Enferm 2002;21:234-9.

4. Gray PH, Trotter JA, Langbridge P, Doherty CV. Pain relief for neonates in Australian hospitals: a need to improve evidence-based practice. J Paediatr Child Health 2006;42:10-3.

5. Simons SH, Tibboel D. Pain perception development and maturation. Semin Fetal Neonatal Med 2006;11:227-31.

6. Grunau R. Early pain in preterm infants. A model of long-term effects. Clin Perinatol 2002;29:373-94.

7. Hohmeister J, Demirakça S, Zohsel K, Flor H, Hermann C. Responses to pain in school-aged children with experience in a neonatal intensive care unit: cognitive aspects and maternal influences. Eur J Pain 2009;13:94-101.

8. Carbajal R, Rousset A, Danan C, Coquery S, Nolent P, Ducrocq S et al. Epidemiology and treatment of painful procedures in neonates in intensive care units. JAMA 2008;300:60-70.

9. Harrison D, Loughnan P, Johnston L. Pain assessment and procedural pain management practices in neonatal units in Australia. J Paediatr Child Health 2006;42:6-9.

10. McKechnie L, Levene M. Procedural pain guidelines for newborn in the United Kingdom. J Perinatol 2008;28:107-11.

11. Prestes AC, Guinsburg R, Balda RC, Marba ST, Rugolo LM, Pachi PR et al. The frequency of pharmacological pain relief in university neonatal intensive care units. J Pediatr (Rio J) 2005;81:405-10.

12. Cignacco E, Hamers JP, van Lingen RA, Zimmermann LJ, Müller R, Gessler $\mathrm{P}$ et al. Pain relief in ventilated preterms during endotracheal suctioning: $\mathrm{a}$ randomized controlled trial. Swiss Med Wkly 2008;138:635-45.

13. Anand KJ, Hall RW, Desai N, Shephard B, Bergqvist LL, Young TE et al. Effects of morphine analgesia in ventilated preterm neonates: primary outcomes from NEOPAIN randomised trial. Lancet 2004;363:1673-82. recursos humanos prejudicam a avaliação e, consequentemente, a minimização da dor ${ }^{(44,46)}$.

Para os neonatologistas, a avaliação da dor deve fazer parte da rotina, durante o exame físico ${ }^{(55)}$. Esta deve ser valorizada como o quinto sinal vital, sendo avaliada de maneira sistematizada e tratada mediante protocolos previamente estabelecidos. É de responsabilidade dos profissionais de saúde, clínicos e pesquisadores, desenvolver, testar e utilizar os melhores métodos possíveis para avaliar a dor em todas as situações potencialmente dolorosas, instituir normatizações nas unidades neonatais e, assim, prover um cuidado apropriado, efetivo e humano para o recém-nascido ${ }^{(20,52,53)}$.

Dessa forma, os estudos evidenciam que, não obstante os avanços no manuseio do processo álgico, há necessidade de melhorias no preparo dos profissionais de saúde acerca da identificação, da avaliação e do controle da dor no período neonatal, na tentativa de modificar as práticas de rotina, esperando fornecer subsídios na busca de uma assistência mais humanizada ao neonato ${ }^{(3,43)}$.

14. Guinsburg R. Assessing and treating pain in the newborn. J Pediatr (Rio J) 1999;75:149-60.

15. Chermont AG, Guinsburg R, Balda RC, Kopelman BI. What do pediatricians know about pain assessment and treatment in newborn infants? J Pediatr (Rio J) 2003;79:265-72.

16. Dodds E. Neonatal procedural pain: a survey of nursing staff. Paediatr Nurs 2003;15:18-21.

17. Silva YP, Gomez RS, Máximo TA, Silva AC. Pain evaluation in neonatology. Rev Bras Anestesiol 2007;57:565-74.

18. Correia LL, Linhares MB. Assessment of the behavior of children in painful situations: literature review. J Pediatr (Rio J) 2008;84:477-86.

19. Anand KJ, Aranda JV, Berde CB, Buckman S, Capparelli EV, Carlo W et al. Summary proceedings from the neonatal pain-control group. Pediatrics 2006;117 (3 Pt 2):S9-22.

20. Scochi CG, Carletti M, Nunes R, Furtado MC, Leite AM. A dor na unidade neonatal sob a perspectiva dos profissionais de enfermagem de um hospital de Ribeirão Preto-SP. Rev Bras Enferm 2006;59:188-94.

21. Herr K, Coyne PJ, Key T, Manworren R, McCaffery M, Merkel S et al. Pain assessment in the nonverbal patient: position statement with clinical practice recommendations. Pain Manag Nurs 2006;7:44-52.

22. Castro MC, Guinsburg R, Almeida MF, Peres CA, Yanaguibashi G, Kopelman BI. Profile of opioid prescriptions for intubated and mechanically ventilated neonates. J Pediatr (Rio J) 2003;79:41-8.

23. Lander J. Clinical judgments in pain management. Pain 1990;42:15-22.

24. Dihle A, Bjølseth $G$, Helseth $S$. The gap between saying and doing in postoperative pain management. J Clin Nurs 2006;15:469-79.

25. Simons SH, van Dijk M, Anand KS, Roofthooft D, van Lingen RA, Tibboel D. Do we still hurt newborn babies? A prospective study of procedural pain and analgesia in neonates. Arch Pediatr Adolesc Med 2003;157:1058-64.

26. Wilson B, McSherry W. A study of nurses' inferences of patients' physical pain J Clin Nurs 2006;15:459-68.

27. Hennig MA, Gomes MA, Gianini NO. Conhecimentos e práticas dos profissionais de saúde sobre a "atenção humanizada ao recém-nascido de baixo peso - método canguru". Rev Bras Saude Mater Infant 2006;6:427-36. 
28. Manias E, Botti M, Bucknall T. Observation of pain assessment and management - the complexities of clinical practice. J Clin Nurs 2002;11:724-33.

29. Craig KD, Pillai RR. Social influences, culture and ethnicity. In: McGrath PJ, Finley GA, editors. Pediatric pain: biological and social context Progress in pain research and management. Seattle (WA): IASP Press Seattle; 2003. p. $159-82$.

30. Breau LM, McGrath PJ, Stevens B, Beyene J, Camfield C, Finley GA et al. Judgments of pain in the neonatal intensive care setting: a survey of direct care staff's perceptions of pain in infants at risk for neurological impairment. Clin J Pain 2006;22:122-9.

31. Hobara M. Beliefs about appropriate pain behavior: cross-cultural and sex differences between Japanese and Euro-Americans. Eur J Pain 2005; 9:389-93.

32. Robinson ME, Wise EA. Prior pain experience: influence on the observation of experimental pain in men and women. J Pain 2004;5:264-9.

33. Guinsburg R, Peres CD, de Almeida MF, Balda RD, Berenguel RC, Tonelotto $\mathrm{J}$ et al. Differences in pain expression between male and female newborn infants. Pain 2000;85:127-33.

34. Gharavi B, Schott C, Nelle M, Reiter G, Linderkamp O. Pain management and the effect of guidelines in neonatal units in Austria, Germany and Switzerland. Pediatr Int 2007;49:652-8.

35. Porter FL, Wolf CM, Gold J, Lotsoff D, Miller JP. Pain and pain management in newborn infants: a survey of physicians and nurses. Pediatrics 1997; 100:626-32.

36. Balda RC, Guinsburg R, de Almeida MF, Peres CA, Miyoshi MH, Kopelman BI. The recognition of facial expression of pain in full-term newborns by parents and health professionals. Arch Pediatr Adolesc Med 2000;154:1009-16.

37. Elias LS, Guinsburg R, Peres CA, Balda RC, Santos AM. Disagreement between parents and health professionals regarding pain intensity in critically ill neonates. J Pediatr (Rio J) 2008;84:35-40.

38. Stevens B, McGrath P, Dupuis A, Gibbins S, Beyene J, Breau L et al. Indicators of pain in neonates at risk for neurological impairment. J Adv Nurs 2009; 65:285-96.

39. Sousa BB, Santos MH, Sousa FG, Gonçalves AP, Paiva SS. Avaliação da dor como instrumento para o cuidar de recém-nascidos pré-termo. Texto Contexto Enferm 2006;15:88-96.

40. Bergqvist LL, Eriksson M, Kronsberg SS, Schollin J, Barton B, Anand KJ. Seeing through the blind! Ability of hospital staff to differentiate morphine from placebo, in neonates at a placebo controlled trial. Acta Paediatr 2007;96:1004-7.

41. Balda RC, Almeida MF, Peres CA, Guinsburg R. Fatores que interferem no reconhecimento por adultos da expressão facial de dor no recém-nascido. Rev Paul Pediatr 2009;27:160-7.

42. Nayak S, Shiflett SC, Eshun S, Levine FM. Culture and gender effects in pain beliefs and the prediction of pain tolerance. Cross Cult Res 2000; 34:135-51.

43. Todd KH, Samaroo N, Hoffman JR. Ethnicity as a risk factor for inadequate emergency department analgesia. JAMA 1993;269:1537-9.

44. Viana DL, Dupas G, Pedreira ML. Children pain assessment by nurses at the Intensive Care Unit. Pediatria (São Paulo) 2006;28:251-61.

45. Pires A. Fatores que afetam a percepção da dor em crianças. In: Silva YP, editor. Dor em pediatria. Rio de Janeiro: Guanabara Koogan; 2006. p. 21-8.

46. Andersen RD, Greve-Isdahl M, Jylli L. The opinion of clinical staff regarding neonatal procedural pain in two Norwegian neonatal intensive care units. Acta Paediatr 2007;96:1000-3.

47. Klopfenstein CE, Herrmann FR, Mamie C, Van Gessel E, Forster A. Pain intensity and pain relief after surgery. A comparison between patients' reported assessments and nurses' and physicians' observations. Acta Anaesthesiol Scand 2000;44:58-62.

48. Anand KJ. Pharmacological approaches to the management of pain in the neonatal intensive care unit. J Perinatol 2007;27:S4-11.

49. Cignacco E, Hamers JP, Stoffel L, van Lingen RA, Gessler P, McDougall J et al. The efficacy of non-pharmacological interventions in the management of procedural pain in preterm and term neonates. A systematic literature review. Eur J Pain 2007;11:139-52.

50. Harvey AJ, Morton NS. Management of procedural pain in children. Arch Dis Child Educ Pract Ed 2007;92:20-6.

51. Bartolomé SM, Cid JL, Freddi N. Analgesia and sedation in children: practical approach for the most frequent situations. J Pediatr (Rio J) 2007;83:S71-82.

52. [No authors listed]. Guideline statement: management of procedure-related pain in neonates. J Paediatr Child Health 2006;42:S31-9.

53. American Academy of Pediatrics Committee on Fetus and Newborn; American Academy of Pediatrics Section on Surgery; Canadian Paediatric Society Fetus and Newborn Committee, Batton DG, Barrington KJ, Wallman C. Prevention and management of pain in the neonate: an update. Pediatrics 2006; 118:2231-41.

54. Heaton P, Herd D, Fernando A. Pain relief for simple procedures in New Zealand neonatal units: practice change over six years. J Paediatr Child Health 2007;43:394-7.

55. Taylor BJ, Robbins JM, Gold JI, Logsdon TR, Bird TM, Anand KJ. Assessing postoperative pain in neonates: a multicenter observational study. Pediatrics 2006;118:e992-1000. 